\title{
Clinical practice guideline for dedicated breast PET
}

\author{
Makoto Hosono - Tsuneo Saga $\cdot$ Kengo Ito $\cdot$ Shinichiro Kumita Masayuki Sasaki $\cdot$ Michio Senda \\ Jun Hatazawa $\cdot$ Hiroshi Watanabe $\cdot$ Hiroshi Ito $\cdot$ Shinichi Kanaya $\cdot$ Yuichi Kimura $\cdot$ Hideo Saji \\ Seishi Jinnouchi $\cdot$ Hiroyoshi Fukukita $\cdot$ Koji Murakami $\cdot$ Seigo Kinuya $\cdot$ Junichi Yamazaki $\cdot$ \\ Mayuki Uchiyama - Koichi Uno - Katsuhiko Kato - Tsuyoshi Kawano · Kazuo Kubota - Takashi Togawa • \\ Norinari Honda $\cdot$ Hirotaka Maruno $\cdot$ Mana Yoshimura $\cdot$ Masami Kawamoto $\cdot$ Yukihiko Ozawa
}

Received: 13 March 2014/ Accepted: 13 March 2014/Published online: 31 May 2014

(C) The Japanese Society of Nuclear Medicine 2014

\section{Introduction}

Dedicated breast positron-emission tomography (dedicated breast PET) is a diagnostic technique using a dedicated breast PET scanner. PET using ${ }^{18}$ F-2-deoxy-2-fluoro-Dglucose (hereafter, FDG) is a very useful diagnostic technique in medical examination. In 2002, FDG-PET was employed for health insurance checks on 12 diseases, mainly cancer. Since breast cancer has been covered under insurance from the beginning, the technique has played an important role, mainly in staging and recurrence screening [1].

According to a report of the National Cancer Center, as regards the probability of survival from breast cancer, there is a significant difference between $\mathrm{T} 1 \mathrm{~b}$ (from 0.5 to less

All authors contributed to the paper as committees' members of Japanese Society of Nuclear Medicine and are listed in the appendix.

M. Hosono $(\bowtie)$

Institute of Advanced Clinical Medicine, Kindai University, 377-2 Ohno-Higashi, Osaka-Sayama, 589-8511 Osaka, Japan e-mail: hosono@med.kindai.ac.jp

M. Hosono - T. Saga $\cdot$ K. Ito $\cdot$ S. Kumita $\cdot$ M. Sasaki · M. Senda $\cdot$ J. Hatazawa $\cdot$ H. Watanabe $\cdot$ H. Ito $\cdot$ S. Kanaya $\cdot$ Y. Kimura · H. Saji · S. Jinnouchi · H. Fukukita · K. Murakami PET Nuclear Medicine Committee, Japanese Society of Nuclear Medicine, Tokyo, Japan

S. Kinuya - J. Yamazaki $\cdot$ M. Uchiyama $\cdot$ K. Uno $\cdot$ K. Kato ·

T. Kawano - K. Kubota - T. Togawa $\cdot$ N. Honda $\cdot$ H. Maruno.

M. Yoshimura

Health Insurance Committee, Japanese Society of Nuclear

Medicine, Tokyo, Japan

M. Kawamoto · Y. Ozawa

Japanese Society of Nuclear Medicine, Tokyo, Japan than $1.0 \mathrm{~cm}$ ) and T1c (from $1.0 \mathrm{~cm}$ to less than $2.0 \mathrm{~cm}$ ) $(P=0.003)$, and it is, therefore, important to detect breast cancer of diameter less than $1.0 \mathrm{~cm}$ [2].

Although mammography is the standard technique for detecting breast cancer, its usefulness is limited in the highdensity mammary gland of young people. On the other hand, although MRI is useful also to detect breast cancer in a high-density mammary gland, and there is a report that its sensitivity and degree of specificity are superior to those of mammography, it is necessary to perform the examination while taking the menstrual cycle into consideration, and there is a concern over the considerable side effects accompanying the use of contrast media.

Although breast cancer is a disease for which health insurance covers diagnosis by FDG-PET, with conventional whole body PET equipment, sensitivity to a small primary tumor of diameter less than $1 \mathrm{~cm}$ is low. The reasons for this are that with whole-body PET, spatial resolution is limited; second, on the image obtained in the dorsal lying position, detectability falls due to respiratory movement; and third, a physiological FDG distribution is also obtained from a healthy mammary gland. Even if it is attempted to obtain an image in the prone lying position or a delayed image, detectability is still insufficient with whole-body PET.

To overcome these problems, a dedicated breast contact PET scanner was developed. Since the breast is located in the body surface, the detector can be brought up close without limitation, and it may be expected that due to the close-up image, spatial resolution would be improved. If respiratory movement can be reduced by fixing the breast, or imaging with the patient in the prone position, it will also lead to an increase of detection sensitivity. It may be expected that by spending time to image only the breast, it will be possible to obtain a detailed image of the 
distribution of FDG in the mammary gland, and the contrast between healthy tissue and diseased tissue will become clear.

The following 2 types of dedicated breast PET exist, including the scanner already approved also in Japan and those under development. We will refer to them temporarily as the opposite type and ring type, according to the placement of detectors.

(1) Opposite type dedicated breast PET scanner

In this type, the breast is interposed between two plate-like detectors to acquire an image, as in the case of a mammography. If the breast is directly fixed, the detector can be brought into close contact, and respiratory movement can be reduced. Tomographic images parallel to the two detectors on either side of the breast can thus be obtained [3-6]. In principle, two images, in the medio-lateral oblique direction and the cranial-caudal direction, are obtained for each of the left and right breasts, i.e., a total of 4 images.

(2) Ring type dedicated breast PET scanner

In this scanner, the detector is in the shape of letter $\mathrm{O}$ or letter C. The breast is suspended at the centre of the detector to acquire the image $[7,8]$.

In Japan also, one type of dedicated breast PET scanner was approved under the Pharmaceutical Affairs Act in August 2012. The dedicated breast PET has about the same sensitivity and specificity as those of mammography or contrast MRI and is regarded as a useful tool to diagnose breast cancer. Although it must be used in conjunction with a conventional whole-body PET, it allows precise staging of the cancer and diagnosis of recurrence and is expected to make a great contribution in deciding future treatment strategies. The Japanese Society of Nuclear Medicine has, therefore, drawn up this guideline to indicate new directions regarding dedicated breast PET so that dedicated breast PET will be used more safely, appropriately, and smoothly. At the present time, dedicated breast PET machines are installed in only a few facilities, but as its use becomes more widespread, clinical data accumulate, and new machines are introduced, the present guideline may be revised.

\section{Dedicated breast PET scanner}

In dedicated breast PET, it is assumed for purposes of health insurance that the scanners used have been approved under the Pharmaceutical Affairs Act and that they will be used in conjunction with whole-body PET.
Inspection of the scanner performance

An inspection must be performed when the scanner is installed, when carrying out large-scale maintenance, or after making adjustments

According to regulations such as the "Directive to prevent accidents when using nuclear medicine diagnostic and therapeutic equipment" drawn up by the Japanese Society of Nuclear Medicine, measurements are performed regarding spatial resolution and uniformity of image density. For this purpose, comparison is made with data supplied by the manufacturer and data for similar scanners published by other facilities.

\section{Pre-operation inspections}

The following inspections must be performed without fail every day before performing examinations:

(1) Overall state of the scanner and operation status.

(2) Confirmation of the state of the detectors.

\section{Regular inspections and adjustments}

(1) Overall state of the scanner and operation status.

(2) Collection of data to correct detector sensitivity.

(3) Correction of uniformity of detectors.

As an example, the following monthly inspections must be performed for the opposite type dedicated breast PET scanner PEM Flex ${ }^{\mathrm{TM}}$, manufactured by Naviscan.

Any discrepancy in the following items from their values at factory shipment:

- Orientation of crystal in the $\mathrm{X}$ and $\mathrm{Y}$ directions.

- Energy peak.

- Energy spectrum.

- Efficiency distribution.

Blank scan.

Test scan with a Ge radiation source.

Scanner maintenance

Refer to "Guideline for performing PET scans using FDG manufactured in the hospital (Vol. 2)" [9], and "Guideline for imaging techniques in FDG-PET scans" [10].

FDG dose and scanning time

The FDG dose is based on a whole-body PET scan.

The FDG dose and scanning time (data collection time) used in a whole-body scan are set so that a suitable 
counting rate and sufficient count are obtained. It is recommended that a dedicated breast PET scan is obtained after performing whole-body PET, and the scanning time must be set taking account of the decay factor particular to the machine.

Image reconstruction and display

There is a default for each type of machine; the image reconstruction technique is determined referring to manufacturer's data and information from other facilities using the same type of machine.

Storage of images and scan result report

Image data are generated as volume data with numerical values. To display this data, it is transferred to the computer of the scanner or another computer and, by using display analysis software, presented as appropriate sections, slices or a MIP (maximum intensity projection) as deemed necessary by the radiologist. Any observations must be described in the form of text, presented in the form of a report, and returned to the doctor requesting the scan. The report is generated and handled in accordance with the "Directive to prevent accidents when using nuclear medicine diagnostic and therapeutic equipment" drawn up by the Japanese Society of Nuclear Medicine".

The original image data must be saved in a digital format which can be retransmitted by the display analysis software. It should be noted that it may not be possible to completely convert PET images to the DICOM standard.

\section{Clinical usage}

\section{Facility criteria}

Since national health insurance stipulates that whole-body PET must be used in conjunction, facilities must satisfy the following criteria regarding calculation of diagnosis and treatment remuneration:

(1) At least 1 regular doctor who has 3 or more years' experience in nuclear medicine diagnosis and has completed the prescribed training.

(2) At least 1 radiological technologist who has specialized knowledge and experience regarding handling of PET pharmaceutical preparations.

A dedicated breast PET scanner is regarded as a single PET device as in the case of whole-body PET, and it is required that there be at least 1 radiological technologist per machine.
Also, because of the radiation that a radiological technologist working with a dedicated breast PET scanner may be exposed to, it is recommended that several radiological technologists work in rotation.

\section{Insurance coverage}

This is based on insurance coverage for breast cancer in PET and PET/CT.

(1) Insurance coverage criteria.

This coverage applies to patients in whom staging, metastasis, and recurrence could not be confirmed by other tests or imaging diagnosis.

(2) Selection criteria for insurance coverage.

(a) Patients in whom a malignant tumor has been confirmed histologically.

(b) Patients in whom, if a definitive diagnosis cannot be obtained histologically, there is clinically a high probability of diagnosing a malignant tumor from the clinical disease picture, bodily symptoms, image diagnostic observations apart from PET or PET/CT, tumor markers, and observations of the clinical course.

\section{Test method}

This is based on the guidelines for conventional wholebody PET.

A dedicated breast PET scan should be obtained quickly after the whole-body PET scan is complete. This is in order to reduce radiation exposure of the radiological technologist when positioning the breast scan, to set a suitable scan range based on the whole-body PET scan, and to achieve an increase of the tumor/background ratio due to passage of time.

In an opposite type scanner, the whole breast is mostly depicted, and images are taken in the medio-lateral oblique direction, and the cranial-caudal direction which complements it.

\section{Determination and diagnosis}

In a dedicated breast PET scan, accumulation sites higher than the background mammary gland are examined by visual evaluation. If possible, it is recommended to refer to a mammography and MRI and perform a comparative interpretation [11].

For a quantitative evaluation, it is first and foremost most desirable to use the SUV (standardized uptake value) which is a quantitative value common to whole-body PET. At the present time, however, it is difficult to interconvert SUV between a whole-body PET scanner and the dedicated breast PET scanner, and therefore a quantitative method specific to dedicated breast PET is now being considered. Methods proposed include the FDG accumulation ratio 
relative to dose per unit body weight, and the accumulation ratio between the lesion area and background mammary gland or mammary gland on the opposite side. In some dedicated breast PET scanners, no absorption correction is made, so it is necessary to take the characteristics of the machine into account.

In FDG-PET, it is difficult to detect malignant tumors with a low grape sugar metabolism, and false negatives sometimes occur. Also, immediately following biopsy, FDG becomes distributed due to inflammation which interferes with a precise diagnosis, so care must be taken. Dedicated breast PET has higher sensitivity and resolution than conventional whole-body PET, but in the case of very small breast cancers, sufficient FDG cannot be accumulated for a diagnosis, so they may be under-estimated.

Precautions in use

\section{FDG dosage}

As with whole-body PET, FDG is administered when it is deemed that its diagnostic usefulness outweighs disadvantage due to radiation exposure. Also, the dosage is kept to the absolute minimum.

\section{Radiation exposure amount}

In general, there is no need to administer an additional dose of FDG in dedicated breast PET scans, so the radiation exposure is similar to that of a whole-body PET scan. In the present guideline, we give a figure based on the report of ICRP Publication 106 [12]. The actual dosage if $185 \mathrm{MBq}$ are administered to an adult is $3.5 \mathrm{mSv}$, and the radiation exposure in a regular transmission scan using ${ }^{68} \mathrm{Ge}^{68} \mathrm{Ga}$ sources is of the order of $0.25 \mathrm{mSv}$. On the other hand, the radiation in a CT scan done for correcting absorption in $\mathrm{PET} / \mathrm{CT}$, with a low radiation scan for generating a fused image, is said to be 1.4-3.5 mSv. If the CT in PET/CT uses a high radiation dose similarly to an ordinary it may be $10 \mathrm{mSv}$ or more, so the need for this must be confirmed.

\section{Precautions regarding dedicated breast PET}

(1) Examination of patients with a pacemaker

For both the opposite type scanner and ring type scanner, lead wires may be broken due to compression of the body surface by the detectors, so due care is required.

(2) Patients who have had surgical implants of foreign materials

In the opposite type scanner, the breast is interposed between the detectors, and as the implant may be damaged by compression, care is required.

(3) Lactating patients
Since FDG accumulates more in the mammary gland tissue during lactation than when not lactating, these patients should not be subjected to a dedicated breast PET scan. In these patients, it is considered undesirable to use FDG, and advised to use it carefully only if it is deemed that the diagnostic benefit outweighs the risk due to radiation exposure.

\section{Application not covered by insurance}

Determining the effect of chemotherapy in progressive breast cancer

The decrease rate of FDG after 2 rounds of chemotherapy in progressive breast cancer is strongly correlated with evaluation of histological therapeutic effect. If reproducibility can be confirmed, it is possible to decide whether to continue the same chemotherapy by performing an FDG-PET scan after completing 2 rounds of chemotherapy or not. In determining the therapeutic effect, dedicated breast PET scanners are expected to have clinical usefulness [13-15].

\section{Screening of high-risk patients}

It is now known that genetics is involved in the increased prevalence of breast cancer and early-onset breast cancer. As genetic breast cancers related to BRCA1 (breast cancer susceptibility gene I) and BRCA2 (breast cancer susceptibility gene II), such as familial breast and ovarian cancer syndrome, occur at an early age, it is recommended that screening be performed earlier than is usually advised. The guideline of the American Cancer Society suggests screening by MRI not only in persons positive for BRCA1/ 2 , but also when the breast cancer lifetime risk exceeds $20 \%$ [16-20]. In recent years, research has progressed. As it is reported that the BRCA genetic mutation rate in Japanese breast cancer/variant cancer families is about the same as that of Europe and the United States, there is a "Guideline for MRI screening in high-risk breast cancer groups" [21]. Dedicated breast PET has about the same diagnostic capability as contrast MRI, and it may become a useful screening technique along with whole-body PET. However, at present, there is not sufficient evidence; hence clinical research should be performed in large numbers of patients in future, and its utility should be evaluated.

\section{Radiation safety}

Basic ideas concerning ensuring radiation safety

Radiation protection from the viewpoint of physiological characteristics, and radiation management in accordance 
with the regulations are based on whole-body PET scans. In medical facilities which perform FDG-PET scans, taking account of social and economic factors, it is important to keep the radiation risk low within realistically achievable limits $[9,22]$.

Radiation safety management system

Based on whole-body PET scans.

Education and training of radiation workers

Based on whole-body PET scans.

Protection of PET scan operators from radiation exposure

The main radiation sources are FDG injectable solution and the patients who receive it, therefore, efforts must be made to reduce external exposure by respecting 3 principles (time, distance and shielding). In a dedicated breast PET scan, unlike a whole-body PET scan, as the radiological technologist must touch the body (mainly the breasts) directly to perform positioning, increased exposure is unavoidable. Therefore, he must be trained to shorten the time required for positioning as far as possible. In a dedicated breast PET scanner which is formed in one piece with a control panel, scanning adjustments are performed in a positron treatment room, and after scanning begins, he should leave the examination room promptly so that he can confirm the progress of the procedure. Also, it is recommended that there be several radiological technologists responsible for dedicated breast PET scans and that they work in rotation.

Measures used to reduce radiation exposure arising from FDG manufacture, FDG injection, and external radiation sources are based on those for a whole-body PET scan.

Instructions and precautions for patients and patient nurses

Based on a whole-body PET scan.

Structural and installation criteria

Based on a whole-body PET scan.

Radiation waste disposal

Based on a whole-body PET scan.

\section{Appendix: Dedicated Breast PET Clinical Practice Guideline Members}

PET Nuclear Medicine Committee Chairperson: Makoto Hosono

Vice-chairperson: Tsuneo Saga

Administrators: Kengo Ito, Shinichiro Kumita, Masayuki Sasaki, Michio Senda, Jun Hatazawa, Hiroshi Watanabe

Committee members: Hiroshi Ito, Shinichi Kanaya, Yuichi Kimura, Hideo Saji, Seishi Jinnouchi, Hiroyoshi Fukukita, Koji Murakami

Health Insurance Committee

Chairperson: Kengo Ito

Vice-chairperson: Shinichiro Kumita

Administrators: Seigo Kinuya, Junichi Yamazaki

Committee members: Mayuki Uchiyama, Koichi Uno, Katsuhiko Kato, Tsuyoshi Kawano, Kazuo Kubota, Takashi Togawa, Norinari Honda, Hirotaka Maruno, Mana Yoshimura

Guideline assistants: Masami Kawamoto, Yukihiko Ozawa

\section{References}

1. FDG-PET, PET/CT Diagnostic Guideline 2012 (Japanese Society of Nuclear Medicine). Kaku Igaku (Jpn. J. Nucl. Med.) 2012;49(4):39-40.

2. Ichizawa $\mathrm{N}$, et al. Long-term results of $\mathrm{T} 1 \mathrm{a}, \mathrm{T} 1 \mathrm{~b}$ and $\mathrm{T} 1 \mathrm{c}$ Invasive breast carcinomas in Japanese women: validation of the UICC T1 subgroup classification. Jpn J Clin Oncol. 2002;32(3): 108-9.

3. Schilling K, et al. The role of positron emission mammography in breast cancer imaging and management. Appl Radiol. 2008; 37(4):26-36.

4. Berg WA, et al. High-resolution fluorodeoxyglucose positron emission tomography with compression ("positron emission mammography") is highly accurate in depicting primary breast cancer. Breast J. 2006;12(4):309-23.

5. Berg WA, et al. Breast cancer: comparative effectiveness of positron emission mammography and MR imaging in presurgical planning for the ipsilateral breast. Radiology. 2011;258(1):59-72.

6. Schilling K, et al. Positron emission mammography in breast cancer presurgical planning: comparisons with magnetic resonance imaging. Eur J Nucl Med Mol Imaging. 2011;38(1):23-36.

7. Furuta M, et al. Basic evaluation of a C-shaped breast PET scanner. IEEE NSS\&MIC 2009 Conference Record 2548-2552.

8. Iima $\mathrm{M}$, et al. Clinical performance of 2 dedicated PET scanners for breast imaging: initial evaluation. J Nucl Med. 2012;53(10): $1534-42$.

9. Guideline for performing PET scans using FDG manufactured in the hospital (Vol. 2) (Japanese Society of Nuclear Medicine) Kaku Igaku (Jpn. J. Nucl. Med.) 2005;42(4):1-22. 
10. Guideline for imaging techniques in FDG-PET scans (Japanese Society of Nuclear Medicine) The Japanese Journal of Nuclear Medicine Technology 2007;27:425-456.

11. Narayanan D, et al. Interpretation of positron emission mammography and MRI by experienced breast imaging radiologists: performance and observer reproducibility. AJR Am J Roentgenol. 2011;196(4):971-81.

12. ICRP Publication 106. Ann ICRP 2008;38.

13. Wahl RL, et al. Metabolic monitoring of breast cancer chemohormonotherapy using positron emission tomography: initial evaluation. J Clin Oncol. 1993;11(11):2101-11.

14. Smith IC, et al. Positron emission tomography using [(18)F]fluorodeoxy-D-glucose to predict the pathologic response of breast cancer to primary chemotherapy. J Clin Oncol. 2000;18(8):1676-88.

15. Rousseau $\mathrm{C}$, et al. Monitoring of early response to neoadjuvant chemotherapy in stage II and III breast cancer by [18F]fluorodeoxyglucose positron emission tomography. J Clin Oncol. 2006;24(34):3566-72.

16. ACR Practice Guideline for the Performance of ContrastEnhanced Magnetic Resonance Imaging (MRI) of the Breast. Breast Imaging and Intervention. 10 http://www.acr.org/QualitySafety/Standards-Guidelines/Practice-Guidelines-by-Modality/
media/ACR/Documents/PGTS/guidelines/MRI_Breast/pdf. Resolution 25. 2008.

17. Mann RM, et al. Breast MRI: guidelines from the European Society of Breast Imaging. Eur Radiol. 2008;18:1307-18.

18. Sugano K, et al. Cross-sectional analysis of germline BRCA1 and BRCA2 mutations in Japanese patients suspected to have hereditary breast/ovarian cancer. Cancer Sci. 2008;99:1967-76.

19. Saslow E, et al. American Cancer Society Guidelines for breast screening with MRI as an adjunct to mammography. Cancer J Clin. 2007;57:75-89.

20. Wang CL, et al. Positron emission mammography: correlation of estrogen receptor, progesterone receptor, and human epidermal growth factor receptor 2 status and 18F-FDG. AJR Am J Roentgenol. 2011;197(2):247-55.

21. Guideline for breast MRI screening in breast cancer high-risk groups, ver.1.0 Japan Association of Breast Cancer Screening, MRI Screening Committee).

22. FDG-PET, PET/CT practice guideline 2012 (Japanese Society of Nuclear Medicine), Kaku Igaku (Jpn. J. Nucl. Med.) 2012; 49(4):391-401. 